David Österle, « Freunde sind wir ja eigentlich nicht ». Hofmannsthal, Schnitzler und das Junge Wien

\title{
Karl Zieger
}

\section{(2) OpenEdition}

1 Journals

\section{Édition électronique}

URL : https://journals.openedition.org/austriaca/1570

DOI : 10.4000 /austriaca. 1570

ISSN : 2729-0603

\section{Éditeur}

Presses universitaires de Rouen et du Havre

\section{Édition imprimée}

Date de publication : 1 juin 2020

Pagination : 266-268

ISBN : 979-10-240-1492-0

ISSN : 0396-4590

\section{Référence électronique}

Karl Zieger, "David Österle, "Freunde sind wir ja eigentlich nicht ». Hofmannsthal, Schnitzler und das Junge Wien », Austriaca [En ligne], 90 | 2020, mis en ligne le 01 juin 2020, consulté le 12 octobre 2022 URL : http://journals.openedition.org/austriaca/1570 ; DOI : https://doi.org/10.4000/austriaca.1570

Ce document a été généré automatiquement le 12 octobre 2022.

Tous droits réservés 


\title{
David Österle, « Freunde sind wir ja eigentlich nicht ». Hofmannsthal, Schnitzler und das Junge Wien
}

\author{
Karl Zieger
}

\section{RÉFÉRENCE}

David Österle, «Freunde sind wir ja eigentlich nicht ». Hofmannsthal, Schnitzler und das Junge Wien, Vienne, Kremayr \& Scheriau, 2019, 224 p., ISBN : 978-3-218-01162-8, $24 €$.

1 C'est un fait incontestable : le groupe d'écrivains rassemblés sous l'étiquette Das Junge Wien (La Jeune Vienne) a joué, dans l'histoire de la littérature, un rôle primordial pour la réputation de la littérature autrichienne - on pourrait même dire pour la prise de conscience, notamment en France, qu'une littérature autrichienne de langue allemande, différente de la littérature allemande elle-même, existe. Cette étiquette est habituellement associée à une autre : celle de «l'esprit viennois fin-de-siècle ", que ce groupe incarne à merveille et qui désigne une période d'effervescence culturelle dans tous les domaines - la « modernité ».

Comme toutes les étiquettes, celle de «La Jeune Vienne» contient une part de vérité : les écrivains ainsi désignés - et, en premier lieu les deux «vedettes » du groupe que sont Hugo von Hofmannsthal et Arthur Schnitzler - représentent effectivement un renouveau de la création littéraire en Autriche dans la dernière décennie du XIX ${ }^{e}$ siècle ; ils intègrent les influences venues de France, des îles Britanniques, de Scandinavie et de Russie dans des créations marquées par leurs expériences «locales", par les crises identitaires qui secouent les esprits en cette fin de siècle, par une attention, voire une sensibilité aiguë pour les « états d'âme ".

Mais s'agit-il pour autant d'un groupe homogène, bien structuré? Loin s'en faut, comme le montre le livre de David Österle, dont le titre est déjà tout un programme et reflète bien l'ambivalence et l'ambiguïté des relations entre les membres de ce groupe : 
«Des amis? Nous ne sommes pas vraiment des amis ». Le titre est, en fait, une citation de l'un des membres les plus importants du groupe, Richard Beer-Hofmann; cette citation comporte pourtant une deuxième partie non négligeable que Österle livre au lecteur (p. 58) : « simplement, nous ne nous tapons pas sur les nerfs, les uns les autres. » Sociologiquement - même si la majorité appartient à la grande bourgeoisie, voire, comme Hofmannsthal, à la noblesse (récente) - et littérairement, cette "Jeune Vienne » est donc loin d'être un bloc; c'est, en fait, Hermann Bahr, son porte-parole auto-désigné, qui l'a présentée comme tel dans un souci de communication tout à fait moderne et dans le but de créer, au sein de la littérature de langue allemande, un contrepoint à Berlin et, dans une moindre mesure, à Munich.

Si l'on peut, tout au long du livre de David Österle, se poser la question de savoir si, oui ou non, Hofmannsthal, Schnitzler, Beer-Hofmann, Salten, Bahr et les autres ont été « des amis » (pour conclure avec Salten que oui, «malgré tout, nous fûmes amis tout au long de notre vie », p. 58), l'auteur montre bien ce qui a été sans doute la force de ce groupe : les réseaux qu'ils ont constitués, dont ils ont finalement mutuellement profité, au-delà de leurs différences...

5 Grâce à une structure essentiellement chronologique qui met alternativement l'accent sur les quatre piliers de "la Jeune Vienne", David Österle offre littéralement une «biographie de groupe ». Il retrace sa vie, des premiers contacts et premiers écrits, des premières rencontres au mythique Café Griensteidl au tout début des années 1890 (et que Stefan Zweig relate d'ailleurs à maintes reprises dans Le Monde d'hier) aux moments de reconnaissance (inter-)nationale et aux grands succès qui les consacrent, à l'approche de la Grande Guerre, comme des écrivains éminents, à fort capital culturel, dans le champ de la littérature de langue allemande. Et si la première décennie du $\mathrm{xx}^{\mathrm{e}}$ siècle accentue aussi une diversification, un certain éloignement réciproque, la découverte de nouveaux horizons, de nouvelles occupations, bref: le fait que chacun d'eux poursuive son propre chemin (le dernier chapitre du livre d'Österle est justement intitulé «Eigene Wege gehen»), ces (anciens) «Jeunes Viennois», et notamment le quatuor Hofmannsthal, Schnitzler, Beer-Hofmannn, Salten, ne se perdent jamais complètement de vue, comme le prouve cette lettre assez touchante de Schnitzler (signée «Ihr A.») à Hofmannsthal (« Mein lieber Hugo») du 9 octobre 1925 qui clôt le livre. Que les deux "amis» se soient vouvoyés encore trente-cinq ans après leur première rencontre n'a rien d'étonnant pour l'époque et, se référant à Olga Schnitzler, Österle révèle (p.74) que l'auteur du Reigen aurait tutoyé un seul de ses confrères... celui qu'il supportait le moins : Hermann Bahr !

Entre les débuts du groupe et son envol vers la reconnaissance, Österle fait revivre, à travers de nombreuses anecdotes, les relations pas toujours faciles ni toujours exemptes de jalousie (plus ou moins mal cachée), d'irritations et de susceptibilités de ses membres. Il est, bien sûr, question de leur relation ambiguë avec Hermann Bahr, dont les changements d'orientation les agacent, lui qui, après les avoir encensés, critique parfois sévèrement l'évolution de ses "amis » (par exemple dans son article «Die Entdeckung der Provinz» avec lequel il inaugure en 1899 sa collaboration au Neues Wiener Tagblatt, un quotidien libéral de grand tirage), mais qui est néanmoins à leurs côtés dans des situations difficiles et défend Schnitzler au moment de la publication de Reigen (1903). Ne manquent pas non plus certains épisodes impliquant Karl Kraus, dont les « Jeunes Viennois » étaient l'une des cibles préférées. 
7 Imbriquant épisodes de la vie personnelle (y compris sentimentale : des grisettes aux femmes célèbres, comme les actrices Adele et Wilhelmine Sandrock ou encore Lou Andreas-Salomé, les "Jeunes Viennois » ont, dans certains cas, visiblement échangé leurs conquêtes), relations amicales et création littéraire, l'auteur évoque les discussions autour du naturalisme (plus présent qu'on ne le pense dans les toutes premières années du groupe) et de l'esthétisme, autour des découvertes freudiennes et autour des différentes orientations littéraires des écrivains. Il relate leurs relations avec les éditeurs (notamment S. Fischer) et avec les directeurs de théatre (par exemple l'importance d'Otto Brahm et du milieu théâtral berlinois par rapport aux réticences de Paul Schlenther, directeur du Burgtheater). Il introduit le lecteur dans les cafés viennois, notamment le « Griensteidl », puis le "Central », dont il montre l'importance pour la constitution du groupe, tout en les démythifiant quant à leur rôle de lieu d'inspiration et de création; il amène le lecteur dans leurs villégiatures (à Ischl et dans le Salzkammergut) et sur les grandes randonnées à vélo qu'entreprennent Schnitzler et Hofmannsthal en Suisse et en Italie.

8 Le livre est bien documenté et soigneusement illustré. Vu le nombre important de personnes évoquées et citées, on regrette cependant l'absence d'un index de noms propres et d'une (vraie) bibliographie, car les références bibliographiques se limitent aux notes regroupées en fin de volume, ce qui rend leur consultation (pendant la lecture) malcommode et, surtout, le repérage des indications bibliographiques intégrales souvent difficile.

9 David Österle a un style alerte, une langue accessible (même aux lecteurs pour qui l'allemand est une langue étrangère). Si son livre ne réserve guère de surprises pour quiconque s'est déjà intéressé à la "modernité viennoise », il représente néanmoins une plaisante et utile synthèse et offre une histoire vivante, riche en épisodes et anecdotes, de ce moment important de la culture autrichienne. 